\title{
Research on social App design
}

\author{
Yannan Wang, \\ North China University of Technology
}

Keywords: social App, design, content, functional, interface

\begin{abstract}
As a social application, social App has a wide user base and has a large market in smartphone application. Therefore, the research on social App design is very important. There are some problems with the design of many social apps, which affect the user experience. A good social App design should be based on full content design, specific functional design and simple interface design.
\end{abstract}

\section{Introduction}

With the wide popularity of smartphones and the rapid development of mobile Internet, mobile phones have been more than just a communication tool. Mobile phone is using more and more widely in the application scope of people's life, work, study, entertainment, communication, etc. Every aspect has been inseparable from the mobile phone. App is short for English Application, App refers to the third party applications of smart phones most of the time.

As mobile intelligent terminal to the function strengthening, multimode, customization and open change the direction of the development platform, the App instead of wap website being the leading mobile phone applications, has become an obvious fact.[1] Although smartphones also brings some problems and troubles, such as some people have a few minutes to see mobile phone will be anxious, some people in the mobile phone battery remnant soon too messy and so on. But what is undeniable is that smartphones represents the development of science and technology, has changed people's life, make people's life, study, work and so on various aspects have become more convenient and more efficient.

According to the 36th statistical report on China's Internet development by CNNIC, the number of Internet users in China reached 0.68 billion 6 by June 2015. Internet penetration is 48.8 percent. The number of mobile Internet users gradually increased to 0.594 billion, up by 36.79 million from December 2014. In addition, the report also showed that entertainment application overall remained stable, with the number of other entertainment applications growing in addition to the decline of online literature users.[2] And social App as social applications software, has a wide range of user groups and occupy the bigger market in terms of smartphone applications, so social App design research also appears very important.

\section{Problems existing in the current social App}

\subsection{Incomplete content}

Social APP belongs to the application of smart phone. It also needs to consider the actual needs of language communication, emotional communication, resource sharing and solving difficulties. Now, some of the mainstream social apps on the market basically around language exchange, emotional communication. It is very limited aspects such as design, ignoring the resource sharing, troubleshoot, etc. to solve the content of the actual demand for the user. And people are faced with some other more specific requirements, such as someone want to sale the goods idle items, someone want to exchange with others idle items, or want unused objects be a gift to continue to play its use value. Pet owners are not able to take care of their pets for many days. They hope to be kept in a neighborhood who have experience in pets. Some people plan to travel but only have to be alone, so they want to find a like-minded companion to travel together. There are also parents who want their children to play with children of the same age. And so on. 


\subsection{The function is not specific}

Many current social App to solve language exchange, emotional communication at the same time, also added some function. Some social App adds a small programs, such as financial deposit, loan, Shared cycling, etc. The increase in many had actually out of the nature of social function, and the function of distribution is not science. It is simply a list together. In this case, users often don't know where to find the entrance when they use the App to solve their own needs, which reduces the use efficiency, affects the user experience feeling, and makes the user experience worse. To sum up, many social apps are not designed to be specific in function. The basic needs of social networking are lost. The demands of many users are not reflected in social apps. Therefore specific design should include a lot of social functions at the beginning of the social App design, such as the game enthusiasts who live in the adjacent communities or in the same community organize online games together. Because searching for objects are in the same community or in the adjacent community, no distance space. This also means that you can establish a fixed time and fixed place offer in order to ensure the continuity of the game. It can quickly build trust, be very convenient to communicate offline game activities, greatly improve game experience to achieve the social function of the App.

\subsection{The interface is not simple enough}

App client interface interaction rules should be accord with the nature of physical motion. Interface is concise and beautiful appearance. Management about the relation between friends, live chat, send and receive private information such as the operation is simple and convenient. The server-side administrator should be able to easily add the recommended information.[3] Good interface design should be based on user experience, convenient for users to use the most concise way to find the information they want, publish their own needs, and be very convenient contact personnel to can solve his needs. The App UI concision will seriously affect the user experience. When user publishing their own needs, or finding how to solve their own needs, they will find a hindrance or take some trouble to find the information they want. This is a direct result of user experience poor feelings or even give up directly using the App. The design of social App interface directly influences the feeling of user experience, and the design of social App should be based on good interface design at the beginning.

\section{How to design a social App}

As mentioned earlier, many current social App content exist insufficient function of comprehensive and specific, the shortcomings of interface concision, which directly affect the user experience. It cannot make social App really play the social function, let the user to solve their own demand, this will lead to the loss of the user. Therefore, the design of good social App should be based on the content design, specific functional design and simple interface design.

\subsection{Comprehensive content design}

The content of the social App design should be based on the demand of people to use the App to solve. It should be truly people-oriented design. The design for everything from people should solve the needs of the people. Overall, the social App on content design should include the following aspects: verbal communication, pet plate, plate game, maintenance plate, secondhand market, tourism plate, food section, car plate, parenting plate, pension plate, etc.

Language communication is the basic content of social App. It should have text, voice, picture, video, positioning and so on. The pet plate is based on a large number of pet lovers. A large proportion of pet lovers are kept pets. When people are accompanied by pets, they need to learn about pet breeding, pet training and pet sports. The pet plate of social App can meet the demand of these pet lovers and pet owners,

Today, social gaming apps are starting to run in the country. Whether it's the number of active users, the size of developers or the revenue from games, you can see that the boom times of social 
gaming are coming. If you compare the traditional online game to the playstation 3 or xbox 360, then the community games are like the Wii, which is a fickle way to rise to the top of the two giants. The community game will play out similar myths soon.[4] Social App can be based on user community or the nearby community. Game players in the social App share the experience and the game experience. People can together with online games, and offline game communication. People can maintain the loyalty of the game to get a better gaming experience through the combination of online and offline.

Now the domestic service industry is on the rise. Sometimes goods are damaged in the home. When contacting the repairman, the service fee of the door-to-door will be more expensive. People can publish maintenance requirements in social App, the man who have maintenance technology and live in the adjacent area can respond to the needs. It is responsible for the residents with maintenance technology can get a income. These who have maintenance requirements can also save part of the expenses.

People also have demands in the travel, food, automobile, child care, pension, etc. The social App including these content can meet the needs of people in these aspects.

\subsection{Specific functional design}

The function of social App implementation should be specific, so that users can quickly solve their own needs.

For example, we can design the functions of children's food, children's reading, children's music, parenting experience, and playmates in the design of the parenting plate. Infant food can share for different ages baby food production, so mom and dad can cook the baby food that is attractive, healthy, nutritious, colourful, lustre and popular. It can help most of the parents solve the problem that the baby doesn't like to eat. Children's reading is also the parents very care about. The parents can share the good reading books, reading methods in a social App. The parents can also meet offline collective reading activities, ant take babies together to read a good book that can have the effect of reading help the baby grow more. Music makes baby's spirit happy and promotes growth. Babies can listen to music suitable for children through social App, so that the baby can grow up happily. At present, although countries opened up a second child, but most of the family is still a baby. A lot of parents don't know how to play with the baby happy on weekends, holidays. With the social App, parents can release requirements. Babies in the adjacent community can be organized together to play, so the problem of the one-child no playmates can be solved.

\subsection{Simple interface design}

Social App interface design should be clear, reasonable layout, concise and lively. The users can quickly solve their own needs. Such as pension sectors designed for the elderly should clearly show the elderly health, elderly sport, elderly tourism, medicare. The elderly users or their children will be able to quickly find how to understand the content, solve their own needs.

In addition, it is also important to consider the concise icon, which is suitable for long, medium, short size and color, font, etc.

\section{4 conclusion}

Good social App design should be based on the comprehensive content design, the specific function design and the simple interface design, which helps to achieve the social function of social App, enhance the user experience, solve the needs of users, access to good reputation, and expand the App of users.

\section{References}

[1] Yapei Zhang, Construction of mobile learning community based on social App, Heilongjiang Science and Technology Information, 2015(16) 
[2] Xinyu Wang, The "golden four hours" of traditional mainstream media, Journalist Cradle, 2011(4)

[3] Song Guo, Design and implementation of the university social system app, Chinascience And Technology Information, 2017(6)

[4] Zhiwei Wen, The new ecology of social network game, Ceocio China, 2010(5) 